\title{
FCGR2A and FCGR3A polymorphisms and clinical outcome in metastatic colorectal cancer patients treated with first-line 5 -fluorouracil/folinic acid and oxaliplatin $+/$ - cetuximab
}

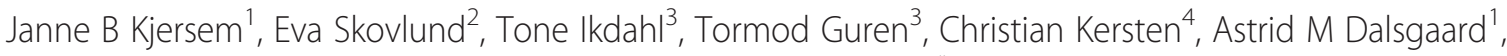 \\ Mette K Yilmaz ${ }^{5}$, Tone Fokstuen ${ }^{6}$, Kjell M Tveit ${ }^{3}$ and Elin H Kure ${ }^{1 *}$
}

\begin{abstract}
Background: Polymorphisms of genes encoding the Fcy receptors (Fc fragment of IgG receptor 2A (FCGR2A) and 3A (FCGR3A)), which influence their affinity for the Fc fragment, have been linked to the pharmacodynamics of monoclonal antibodies. Most studies have been limited by small samples sizes and have reported inconsistent associations between the FCGR2A and the FCGR3A polymorphisms and clinical outcome in metastatic colorectal cancer (mCRC) patients treated with cetuximab. We investigated the association of these polymorphisms and clinical outcome in a large cohort of mCRC patients treated with first-line 5-fluorouracil/folinic acid and oxaliplatin (Nordic FLOX) +/- cetuximab in the NORDIC-VII study (NCT00145314).
\end{abstract}

Methods: 504 and 497 mCRC patients were evaluable for the FCGR2A and FCGR3A genotyping, respectively. Genotyping was performed on TaqMan ABI HT 7900 (Applied Biosystems, Foster City, CA, USA) with pre-designed SNP genotyping assays for FCGR2A (rs1801274) and FCGR3A (rs396991).

Results: The response rate for patients with the FCGR2A R/R genotype was significantly increased when cetuximab was added to Nordic FLOX (31\% versus 53\%, interaction $P=0.03$ ), but was not significantly different compared to the response rate of patients with the FCGR2A H/H or H/R genotypes given the same treatment. A larger increase in response rate with the addition of cetuximab to Nordic FLOX in patients with KRAS mutated tumors and the FCGR2A R/R genotype was observed (19\% versus 50\%, interaction $P=0.04$ ). None of the FCGR3A polymorphisms were associated with altered response when cetuximab was added to Nordic FLOX (interaction $P=0.63$ ). Neither of the FCGR polymorphisms showed any significant associations with progression-free survival or overall survival.

Conclusion: Patients with KRAS mutated tumors and the FCGR2A R/R polymorphism responded poorly when treated with chemotherapy only, and experienced the most benefit of the addition of cetuximab in terms of response rate.

Keywords: Colorectal cancer, FCGR2A, FCGR3A, Polymorphism, Cetuximab

\section{Background}

The prognosis for patients with metastatic colorectal cancer (mCRC) remains poor even though the addition of newer chemotherapeutic agents and targeted drugs has increased the median survival from 12 months with fluorouracil monotherapy to roughly 2 years [1].

\footnotetext{
* Correspondence: Elin.Kure@rr-research.no

'Department of Genetics, Institute for Cancer Research, Oslo University Hospital, Postboks 4953 Nydalen, 0424 Oslo, Norway

Full list of author information is available at the end of the article
}

Cetuximab, a monoclonal antibody targeting the epidermal growth factor receptor (EGFR), has shown efficacy in combination with chemotherapy or given as monotherapy in a small fraction of mCRC patients [2]. Clinical benefit seems to be restricted to patients with KRAS wild-type tumors [3,4]. In the recent NORDIC-VII study, however, we did not find an improved outcome of adding cetuximab to first-line oxaliplatin-based chemotherapy in patients with KRAS wild-type tumors [5]. Similar results were found by the COIN trial and the recent EPOC study $[6,7]$. The 
results of these trials demonstrate the necessity to explore predictive markers independent of KRAS status to avoid unnecessary drug toxicity and reduce treatment cost.

Cetuximab may exert its antitumor effect through multiple mechanisms. One mechanism of its antitumor effects is through antibody-dependent cellular cytotoxicity (ADCC) [8]. ADCC is induced through the interaction of the Fc region of the monoclonal antibody with the Fc gamma receptor (FCGR), surface receptors for immunoglobulin G (IgG), located on immune effector cells such as natural killer lymphocytes and macrophages [9]. Polymorphisms have been demonstrated on genes encoding for the receptors FCGR2A and FCGR3A, affecting their affinity to human IgG: a histidine(H)/arginine( $\mathrm{R})$ polymorphism at position 131 for FCGR2A and a valine (V)/phenylalanine (F) polymorphism at position 158 for FCGR3A [10]. The polymorphisms have been reported to be associated with clinical outcome to the monoclonal antibodies rituximab $[11,12]$ and trastuzumab $[13,14]$ in the treatment of lymphoma and breast cancer, respectively.

Previous studies exploring these polymorphisms in relation to cetuximab effect in mCRC have shown conflicting results and have been dominated by low-powered studies. The aim of the present study was to investigate the association between these polymorphisms and the effect of cetuximab treatment in a large $\mathrm{mCRC}$ patient cohort; the NORDIC-VII cohort. We examined the FCGR2A and FCGR3A polymorphisms as potential markers to predict cetuximab effect in 504 and 497 evaluable mCRC patients, respectively, treated with conventional chemotherapy (Nordic FLOX) with and without the addition of cetuximab.

\section{Methods NORDIC VII}

In the NORDIC VII trial (NCT00145314, registered September 2, 2005), a total of 571 patients with mCRC were randomized to receive first-line standard Nordic FLOX (bolus 5-fluorouracil/folinic acid and oxaliplatin) (arm A), cetuximab and Nordic FLOX (arm B), or cetuximab combined with intermittent Nordic FLOX (arm C). Primary endpoint was progression-free survival (PFS). Overall survival (OS) and response rate were secondary endpoints. DNA from primary tumors was screened for the presence of seven KRAS mutations (codons 12 (G12D, G12A, G12V, G12S, G12C, G12R) and $13(\mathrm{G} 13 \mathrm{D})$ ) and one BRAF (BRAF V600E) mutation as previously described [5]. KRAS and BRAF mutation analyses were obtained in $498(88 \%)$ and 457 patients (81\%), respectively. KRAS mutations in codons 12 and 13 were found in $39 \%$ of the tumors. BRAF mutations (V600E) were present in $12 \%$ of the tumors. The mutational frequencies of the 195 KRAS mutations in the
NORDIC VII cohort were; G12A (9.7\%), G12R (1.5\%), G12D (35.4\%), G12C (9.7\%), G12S (6.2\%), G12V (15.4\%), and G13D (22.1\%). Cetuximab did not add significant benefit to Nordic FLOX and KRAS mutation was not predictive for cetuximab effect. DNA from a total of 504 and 497 of the 566 patients in the intention to treat population was evaluable for the FCGR2A and FCGR3A genotyping, respectively. There were 172 patients in arm A and 332 patients in arms $\mathrm{B}$ and $\mathrm{C}$ evaluable for response and survival analyses for the FCGR2A polymorphism. There were 169 patients in arm A and 328 patients in arms B and C evaluable for response and survival analyses for the FCGR3A polymorphism. KRAS status was available from 442 and 437 patients with FCGR2A and FCGR3A status, respectively. $B R A F$ status was available from 410 and 405 patients with FCGR2A and FCGR3A status, respectively. Response status was evaluated according to the RECIST version 1.0 criteria and was assigned to patients with complete or partial remission with changes in tumor measurements confirmed by repeat studies performed no less than 4 weeks after the criteria for response were first met (minimal interval of 8 weeks -4 cycles) [15]. The study was approved by national ethics committees and governmental authorities in each country and was conducted in accordance with the Declaration of Helsinki. All patients provided written informed consent.

Primary tumors in the NORDIC VII study were screened for KRAS exon 2 (codons 12 and 13) mutations. However, recent studies have demonstrated that wild-type $R A S$ should be defined by the absence of KRAS exons 2, 3, and 4 mutations and the absence of NRAS exons 2, 3, and 4 mutations [16-18]. A follow-up study of the NORDIC VII cohort will include these additional mutational analyses.

\section{FCGR2A-H131R and FCGR3A-V158F genotyping}

Genotyping was performed on a TaqMan ABI HT 7900 (Applied Biosystems, Foster City, CA, USA) with predesigned SNP genotyping assays for FCGR2A c.535A > G (rs1801274; resulting in amino-acid change of histidine to arginine at position 131) and FCGR3A c.818A > C (rs396991; resulting in amino-acid change of valine to phenylalanine at position 158), according to the manufacturer's protocol. Negative controls (water) were included.

\section{Statistical analyses}

The $\chi^{2}$-test and one-way ANOVA were used to compare categorical and continuous variables between groups, as appropriate, respectively. Homoscedasticity was ascertained and the non-parametric Kruskal-Wallis test was applied as a sensitivity analysis. For the prognostic analyses all three arms (arms A, B and C) were analyzed together. For the predictive analyses of cetuximab effect by FCGR2A or FCGR3A genotype, arm A was compared to arms $\mathrm{B}$ and $\mathrm{C}$ combined. The associations between the FCGR2A and 
FCGR3A genotypes and tumor response were analyzed by binary logistic regression. PFS and OS times were estimated using the Kaplan-Meier method. The associations of the FCGR2A and FCGR3A genotypes and PFS and OS were analyzed by Cox's proportional hazards model. The assumption of proportional hazards was checked by inspection of $\log$ minus log plots. The potential value of FCGR2A and FCGR3A as predictive markers of cetuximab effect was analyzed by including an interaction term in the models. The distributions of the FCGR $2 A$ and FCGR3A genotypes in the NORDIC-VII study were tested for Hardy-Weinberg equilibrium [19]. $P<0.05$ was considered statistically significant. All statistical analyses were performed using Statistical Package for Social Sciences, version 18.0 (SPSS Chicago, IL).

\section{Results}

Patient characteristics

Table 1 depicts the frequencies of the analyzed FCGR2A and FCGR3A genotypes, which were in Hardy-Weinberg equilibrium $(P=0.41$ and 0.54 , respectively). There were no significant associations of any of the FCGR2A or FCGR3A genotypes with clinicopathological characteristics (age, sex, location of primary tumor, metastatic sites, $K R A S$, or BRAF mutation status) or treatment, Table 2.

\section{Response rate and survival}

There was no significant difference in response rates for the different FCGR2A and FCGR3A genotypes when analyzing all the three treatment arms together $(P=0.89$ and 0.82 , respectively), Table 2 . There was also no significant association of any of the FCGR2A or FCGR3A genotypes with PFS (Log rank $P=0.45$ and 0.76 , respectively) or OS (Log rank $P=0.42$ and 0.77 , respectively), Table 2 .

\section{Predictive analyses for benefit of cetuximab treatment}

The FCGR2A R/R genotype was associated with increased response rate when cetuximab was added to Nordic FLOX regardless of mutational status (31\% in arm A versus 53\% in arms $\mathrm{B}+\mathrm{C}$, interaction $P=0.03$ ), but was not significantly different compared to the response rate of patients with the $F C G R 2 A \mathrm{H} / \mathrm{H}$ or $\mathrm{H} / \mathrm{R}$ genotypes given the same treatment, Table 3 and Figure 1. There was no significant difference in response rates in the FCGR2A subgroups in patients with $K R A S$ wild-type tumors after the addition of cetuximab, Table 4 and Figure 2. A significant increase in response rate with the addition of cetuximab to Nordic FLOX in patients with KRAS mutated tumors and the FCGR2A $R / R$ genotype was observed (19\% versus $50 \%$, interaction $P=0.04$ ), Table 4 and Figure 3. None of the FCGR3A polymorphisms were associated with altered response when cetuximab was added to Nordic FLOX (interaction $P=0.63$ ), Table 3 . The FCGR3A genotypes were not associated with response to cetuximab when stratified for $B R A F$ or $K R A S$ mutational status, Table 5.

Median PFS and OS were similar in arms $\mathrm{B}+\mathrm{C}$ as compared to arm A for the FCGR2A (Log rank $P=0.35$ and 0.85$)$ and the FCGR3A (Log rank $P=0.41$ and 0.78 ) genotypes, Table 3 . The median PFS and OS were also similar in arms $\mathrm{B}+\mathrm{C}$ compared to arm $\mathrm{A}$ for both the FCGR2A and FCGR3A genotypes when stratified for $B R A F$ or KRAS mutational status, Tables 4 and 5 .

\section{Discussion}

We studied the FCGR2A and the FCGR3A polymorphisms in a large cohort of $\mathrm{mCRC}$ patients treated with conventional chemotherapy with and without cetuximab in an effort to explore potential associations between these polymorphisms and cetuximab effect. Our results show that the addition of cetuximab to Nordic FLOX lead to a statistically significant increase in response rate in patients with the FCGR $2 A \mathrm{R} / \mathrm{R}$ genotype. Subgroup analysis of patients with $K R A S$ mutated tumors and the $F C G R 2 A \mathrm{R} / \mathrm{R}$ genotype showed an even larger increase in response after the addition of cetuximab.

Previous studies exploring the relation between the FCGR polymorphisms and cetuximab efficacy in mCRC have demonstrated conflicting or negative results and have been mostly low-powered studies with small sample sizes. Our study is one of the largest reported so far

Table 1 FCGR2A and FCGR3A genotypes in the study population

\begin{tabular}{llll}
\hline & $\begin{array}{l}\text { Actual frequency } \\
\text { of genotypes }(\mathbf{n})\end{array}$ & $\begin{array}{l}\text { Expected frequency } \\
\text { of genotypes }(\mathbf{n})\end{array}$ & $\begin{array}{l}\text { Hardy-Weinberg equilibrium } \\
\boldsymbol{X}^{2} \text { (1 degree of freedom) }\end{array}$ \\
\hline FCGR2A & & & 0.68 \\
\hline $\mathrm{H} / \mathrm{H}$ & 114 & 118.61 & \\
\hline $\mathrm{H} / \mathrm{R}$ & 261 & 251.78 & \\
\hline $\mathrm{R} / \mathrm{R}$ & 129 & 133.61 & 0.41 \\
\hline FCGR3A & & & \\
\hline F/F & 241 & 238.10 & \\
\hline FN & 206 & 211.8 & \\
\hline VN & 50 & 47.10 & \\
\hline
\end{tabular}


Table 2 Patient characteristics and treatment outcome by FCGR2A and FCGR3A genotypes

\begin{tabular}{|c|c|c|c|c|c|c|c|c|}
\hline & \multicolumn{4}{|l|}{ FCGR2A } & \multicolumn{4}{|l|}{ FCGR3A } \\
\hline & $H / H$ & $H / R$ & $R / R$ & $P$-value & $F / F$ & $F / V$ & $V / V$ & $P$-value \\
\hline Number of patients (\%) & $114(22.6 \%)$ & $261(51.8 \%)$ & $129(25.6 \%)$ & & $241(48.5 \%)$ & $206(41.4 \%)$ & $50(10.1 \%)$ & \\
\hline Age, median (range) & $61(27-74)$ & $62(24-75)$ & $62(30-75)$ & $0.99^{*}$ & $62(24-75)$ & $61(29-75)$ & $61(35-75)$ & $0.47^{*}$ \\
\hline Sex, female/male & $49 / 65$ & $102 / 159$ & $57 / 72$ & $0.58^{+}$ & $93 / 148$ & $94 / 112$ & $20 / 30$ & $0.32^{\dagger}$ \\
\hline Location, colon/rectum & $71 / 43$ & $145 / 116$ & $78 / 51$ & $0.41^{\dagger}$ & $147 / 94$ & $121 / 85$ & $23 / 27$ & $0.15^{\dagger}$ \\
\hline Metastatic sites, 1/>1 & $32 / 82$ & $63 / 198$ & $39 / 90$ & $0.41^{\dagger}$ & $62 / 179$ & $51 / 155$ & $20 / 30$ & $0.08^{\dagger}$ \\
\hline KRAS, wt/mutated & $62 / 38$ & $150 / 82$ & $61 / 49$ & $0.26^{+}$ & $126 / 87$ & $111 / 67$ & $31 / 15$ & $0.54^{\dagger}$ \\
\hline BRAF, wt/mutated & $80 / 11$ & $192 / 26$ & $91 / 10$ & $0.88^{+}$ & $182 / 18$ & $141 / 21$ & $36 / 7$ & $0.28^{+}$ \\
\hline Treatment, FLOX/FLOX + cetuximab & $33 / 81$ & $90 / 171$ & $49 / 80$ & $0.34^{\dagger}$ & $79 / 162$ & $75 / 131$ & $15 / 35$ & $0.58^{\dagger}$ \\
\hline Response; response/no-response & $54 / 60$ & $121 / 140$ & $57 / 72$ & $0.89^{\dagger}$ & $109 / 132$ & $93 / 113$ & $25 / 25$ & $0.82^{\dagger}$ \\
\hline PFS (months), median & 8.3 & 7.9 & 7.6 & $0.45^{\ddagger}$ & 7.9 & 7.6 & 8.4 & $0.76^{\ddagger}$ \\
\hline OS (months), median & 21.9 & 19.8 & 18.2 & $0.42^{\ddagger}$ & 19.9 & 20.5 & 19.7 & $0.77^{\ddagger}$ \\
\hline
\end{tabular}

${ }^{\text {*} O n e-w a y ~ A N O V A ~(T h e ~ K r u s k a l-W a l l i s ~ t e s t ~ p r o d u c e d ~ s i m i l a r ~ p-v a l u e s), ~}{ }^{\dagger}$ Chi-square test ${ }^{\ddagger}$ Log-rank test.

and unlike most of the other studies we included a control group where patients did not receive cetuximab.

Even though their results were not statistically significant, the FCGR2A R/R genotype had a better response rate compared to the $\mathrm{H} / \mathrm{R}$ or the $\mathrm{H} / \mathrm{H}$ genotypes in $K R A S$ wildtype patients treated with cetuximab or panitumumab as monotherapy or in combination with chemotherapy in a study of 104 refractory mCRC patients [20]. Furthermore, a pooled analysis including $217 \mathrm{mCRC}$ patients treated with cetuximab alone or with chemotherapy showed that patients with the $F C G R 2 A$ R/R or H/R alleles had a statistically significant longer median PFS than the $\mathrm{H} / \mathrm{H}$ genotype [21]. Moreover, a study by Negri et al., where most of the 86 mCRC patients enrolled in the study were treated with cetuximab and irinotecan, demonstrated a higher OS in mCRC patients with the FCGR2A R/R polymorphism [22]. However, the authors concluded that the polymorphism was not predictive of cetuximab effect since no relation to response or time to progression (TTP) was demonstrated [22].
Conversely, a study which included $69 \mathrm{mCRC}$ patients reported the $F C G R 2 A \mathrm{H} / \mathrm{H}$ alone or in combination with $F C G R 3 A \mathrm{~V} / \mathrm{V}$ to be associated with longer PFS in irinotecan-refractory mCRC patients with KRAS wildtype and KRAS mutated tumors treated with cetuximab plus irinotecan [23]. The difference remained significant for KRAS mutated patients. Similar results were demonstrated by Rodriguez et al., who reported that patients with any FCGR2A $\mathrm{H}$ and/or FCGR3A V allele were more likely to show a response or have stable disease [24]. Rodriguez et al. explored if the FCGR genotypes would predict which patients with a KRAS, or other downstream mutations, would respond to cetuximab. They included $47 \mathrm{mCRC}$ patients treated with cetuximab and standard chemotherapy with a KRAS, BRAF, NRAS, or PI3K mutation in the FCGR genotype analysis. Two other studies including 52 and $49 \mathrm{mCRC}$ patients, respectively, reported only the FCGR3A V/V genotype to be associated with a better response to cetuximab $[25,26]$.

Table 3 Treatment outcome by FCGR2A and FCGR3A genotypes, and therapy received

\begin{tabular}{|c|c|c|c|c|c|c|c|}
\hline \multirow[b]{2}{*}{ FCGR2A } & \multicolumn{3}{|l|}{ FLOX } & \multicolumn{3}{|c|}{ FLOX + cetuximab } & \multirow[t]{2}{*}{ Interaction $P$-value } \\
\hline & $\overline{H / H}$ & $H / R$ & $R / R$ & $\overline{H / H}$ & $H / R$ & $R / R$ & \\
\hline Number of patients & $N=33$ & $N=90$ & $N=49$ & $N=81$ & $N=171$ & $N=80$ & \\
\hline Response (\%) & $58 \%(19 / 33)$ & $41 \%(37 / 90)$ & $31 \%(15 / 49)$ & $43 \%(35 / 81)$ & $49 \%(84 / 171)$ & $53 \%(42 / 80)$ & $0.03^{*}$ \\
\hline PFS, median (months) & 8.4 & 7.9 & 7.5 & 8.3 & 7.8 & 7.6 & $0.35^{\dagger}$ \\
\hline OS, median (months) & 28.0 & 20.5 & 19.8 & 21.4 & 19.5 & 17.3 & $0.85^{\dagger}$ \\
\hline FCGR3A & $F / F$ & $F / V$ & $V / V$ & $F / F$ & $F / V$ & $V / V$ & \\
\hline Number of patients & $N=79$ & $N=75$ & $N=15$ & $N=162$ & $N=131$ & $N=35$ & \\
\hline Response (\%) & $38 \%(30 / 79)$ & $41 \%(31 / 75)$ & $53 \%(8 / 15)$ & $49 \%(79 / 162)$ & $47 \%(62 / 131)$ & $49 \%(17 / 35)$ & $0.63^{*}$ \\
\hline PFS, median (months) & 7.6 & 8.4 & 7.8 & 8.1 & 7.4 & 9.3 & $0.41^{\dagger}$ \\
\hline OS, median (months) & 20.4 & 20.5 & 19.7 & 19.7 & 21.1 & 20.1 & $0.78^{+}$ \\
\hline
\end{tabular}

${ }^{*}$ Logistic regression, ${ }^{\dagger}$ Cox proportional hazard model. 


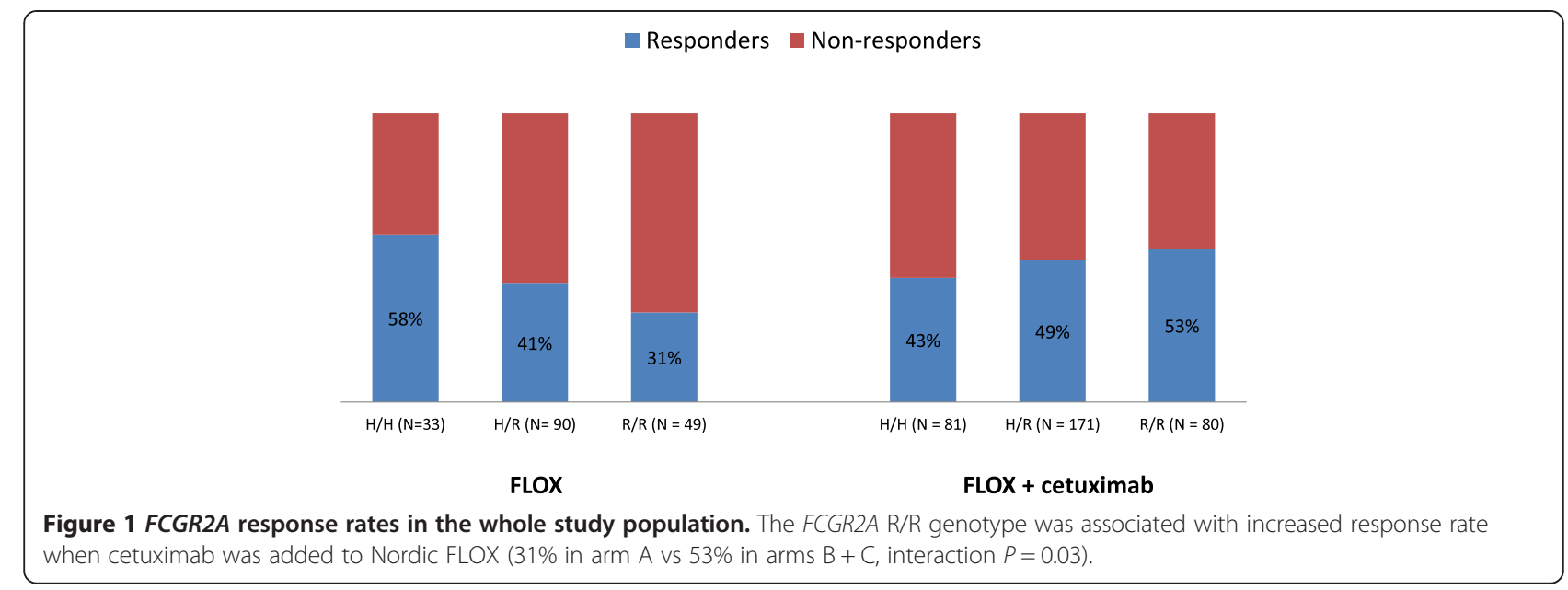

In contrast, three other studies including 65,58 , and 122 mCRC patients, respectively, have reported the FCGR3A F/F allele to be associated with a better clinical outcome [27-29]. The former study demonstrated that patients enrolled in the BOND-2 study with the FCGR3A $\mathrm{F} / \mathrm{F}$ allele had a significantly better response to cetuximab in combination with bevacizumab in irinotecan-refractory mCRC patients [27]. There was shorter survival in patients with the FCGR3A V/V genotype as compared to $\mathrm{V} / \mathrm{F}$ or $\mathrm{F} / \mathrm{F}$ in the study of $58 \mathrm{mCRC}$ patients who received irinotecan in combination with cetuximab [28].
This was shown in the whole study population and in a subgroup analysis of patients with KRAS wild-type tumors. Moreover, the latter study by Pander et al., found mCRC patients in the CAIRO2 study with the FCGR3A F/F allele to be associated with longer PFS in KRAS wildtype patients treated with cetuximab as first-line treatment in combination with capecitabine, oxaliplatin and bevacizumab [29]. A smaller study including only $39 \mathrm{mCRC}$ patients reported the FCGR2A, any $\mathrm{H}$ allele, and FCGR3A, any $\mathrm{F}$ allele, to be associated with longer PFS in mCRC patients who were treated with single-agent cetuximab

Table 4 Treatment outcome by FCGR2A genotype, KRAS or BRAF mutational status, and therapy received

\begin{tabular}{|c|c|c|c|c|c|c|c|}
\hline & \multicolumn{3}{|l|}{ FLOX } & \multicolumn{3}{|c|}{ FLOX + cetuximab } & \multirow[b]{2}{*}{ Interaction $P$-value } \\
\hline & $H / H$ & $H / R$ & $R / R$ & $H / H$ & $H / R$ & $R / R$ & \\
\hline \multicolumn{8}{|c|}{ KRAS wild-type $(\mathrm{N}=273)$} \\
\hline Number of patients & $N=16$ & $N=52$ & $N=20$ & $N=46$ & $N=98$ & $N=41$ & \\
\hline Response & $63 \%(10 / 16)$ & $42 \%(22 / 52)$ & $45 \%(9 / 20)$ & $46 \%(21 / 46)$ & $51 \%(50 / 98)$ & $56 \%(23 / 41)$ & $0.27^{*}$ \\
\hline PFS & 8.4 & 8.9 & 9.0 & 7.7 & 7.7 & 8.0 & $0.23^{+}$ \\
\hline OS & 31.6 & 23.6 & 19.0 & 21.4 & 20.7 & 18.9 & $0.23^{+}$ \\
\hline \multicolumn{8}{|c|}{ KRAS mutated $(\mathrm{N}=169)$} \\
\hline Number of patients & $N=10$ & $N=25$ & $N=21$ & $N=28$ & $N=57$ & $N=28$ & \\
\hline Response & $60 \%(6 / 10)$ & $52 \%(13 / 25)$ & $19 \%(4 / 21)$ & $36 \%(10 / 28)$ & $46 \%(26 / 57)$ & $50 \%(14 / 28)$ & $0.04^{*}$ \\
\hline PFS & 8.1 & 8.3 & 7.1 & 7.7 & 8.1 & 6.7 & $0.90^{+}$ \\
\hline OS & 17.2 & 20.4 & 24.3 & 21.1 & 20.0 & 16.8 & $0.34^{\dagger}$ \\
\hline \multicolumn{8}{|c|}{ BRAF wild-type $(\mathrm{N}=363)$} \\
\hline Number of patients & $N=22$ & $N=62$ & $N=34$ & $N=58$ & $N=130$ & $N=57$ & \\
\hline Response & $64 \%(14 / 22)$ & $48 \%(30 / 62)$ & $35 \%(12 / 34)$ & $47 \%(27 / 58)$ & $52 \%(68 / 130)$ & $54 \%(31 / 57)$ & $0.10^{*}$ \\
\hline PFS & 9.3 & 8.9 & 7.7 & 8.5 & 8.1 & 8.0 & $0.47^{\dagger}$ \\
\hline OS & 31.6 & 23.8 & 21.5 & 21.9 & 21.5 & 17.6 & $0.93^{+}$ \\
\hline \multicolumn{8}{|c|}{$B R A F$ mutated $(\mathrm{N}=47)$} \\
\hline Number of patients & $N=3$ & $N=10$ & $N=4$ & $N=8$ & $N=16$ & $N=6$ & \\
\hline Response & $33 \%(1 / 3)$ & $20 \%(2 / 10)$ & $0 \%(0 / 4)$ & $13 \%(1 / 8)$ & $25 \%(4 / 16)$ & $33 \%(2 / 6)$ & $0.72^{*}$ \\
\hline PFS & 4.3 & 5.1 & 3.8 & 3.8 & 4.6 & 5.8 & $0.36^{+}$ \\
\hline OS & 9.2 & 9.4 & 5.6 & 8.9 & 8.1 & 11.3 & $0.73^{+}$ \\
\hline
\end{tabular}

${ }^{*}$ Logistic regression, ${ }^{\dagger}$ Cox proportional hazard model. 


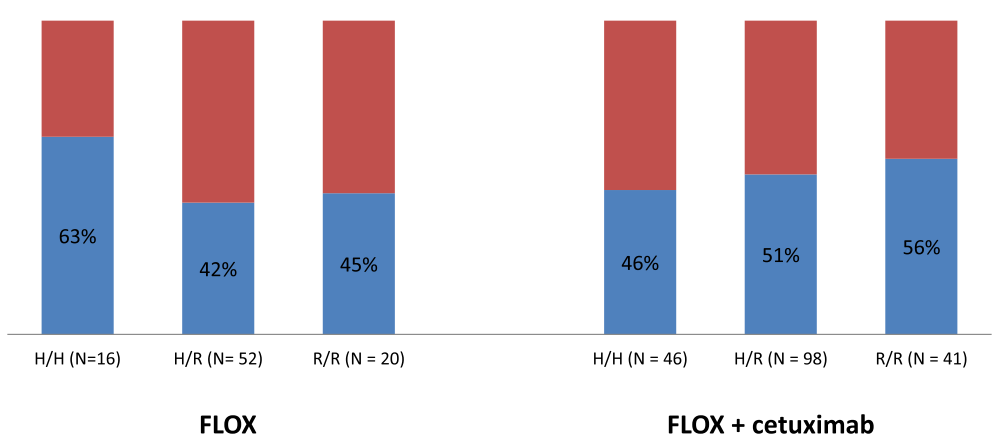

Figure 2 FCGR2A response rates in patients with KRAS wild-type tumors. There was no significant difference in response rates when cetuximab was added to Nordic FLOX in the different FCGR2A subgroups (interaction $P=0.27$ ).

[30]. These results could though not be replicated when the sample size was increased to a total of 130 patients [31]. In addition to the study by Lurje et al., four other studies with a higher number of patients have reported lack of significant associations of the FCGR2A or FCGR3A polymorphisms and cetuximab efficacy in mCRC [20,32-34].

Our study show that patients with KRAS mutated tumors and the FCGR2A R/R genotype responded poorly when treated with chemotherapy only and experienced the most benefit of the addition of cetuximab in terms of response rate. In line with this, Correale et al. demonstrated that activating KRAS mutations in colon cancer cell lines may correlate with a higher susceptibility to cetuximab-mediated ADCC [35]. Another study by Schlaeth et al. found that KRAS mutated tumor cells could be effectively killed by ADCC, indicating that mutated $K R A S$ is not enough to confer resistance to antibodymediated cell killing [36].

The conflicting findings in the different studies demonstrate the importance of sample size when studying the effect of polymorphisms in relation to clinical outcome. Moreover, the heterogeneity among the different studies, such as study design, ethnicity, previous and concomitant treatment, and the distribution of genotypes may also partly explain the discordance. Furthermore, the retrospective nature of most of the studies and the use of different endpoints may also contribute to the conflicting results. Additionally, Clynes et al. found the IgG1 antibodies trastuzumab and rituximab to engage in both activatory (FCGR3A) and inhibitory receptors (FCGR2B) and the in vivo activity of the antibodies may be more predictable by the ratio of FCGR3A to FCGR2B (A/I ratio) [37] which has not been investigated in the reported studies. Furthermore, all the studies have only tested two polymorphisms in only two genes involved in the ADCC mechanism. Also, other effector mechanisms of cetuximab may play a more important role, such as complement-dependent cytotoxicity, apoptosis and phagocytosis.

More importantly, ADCC may not play a correspondingly important role in metastatic cancer patients as demonstrated in in vitro models. ADCC has been shown to be markedly impaired with natural killer cell dysfunction in cancer patients with metastatic disease [38]. Moreover, the

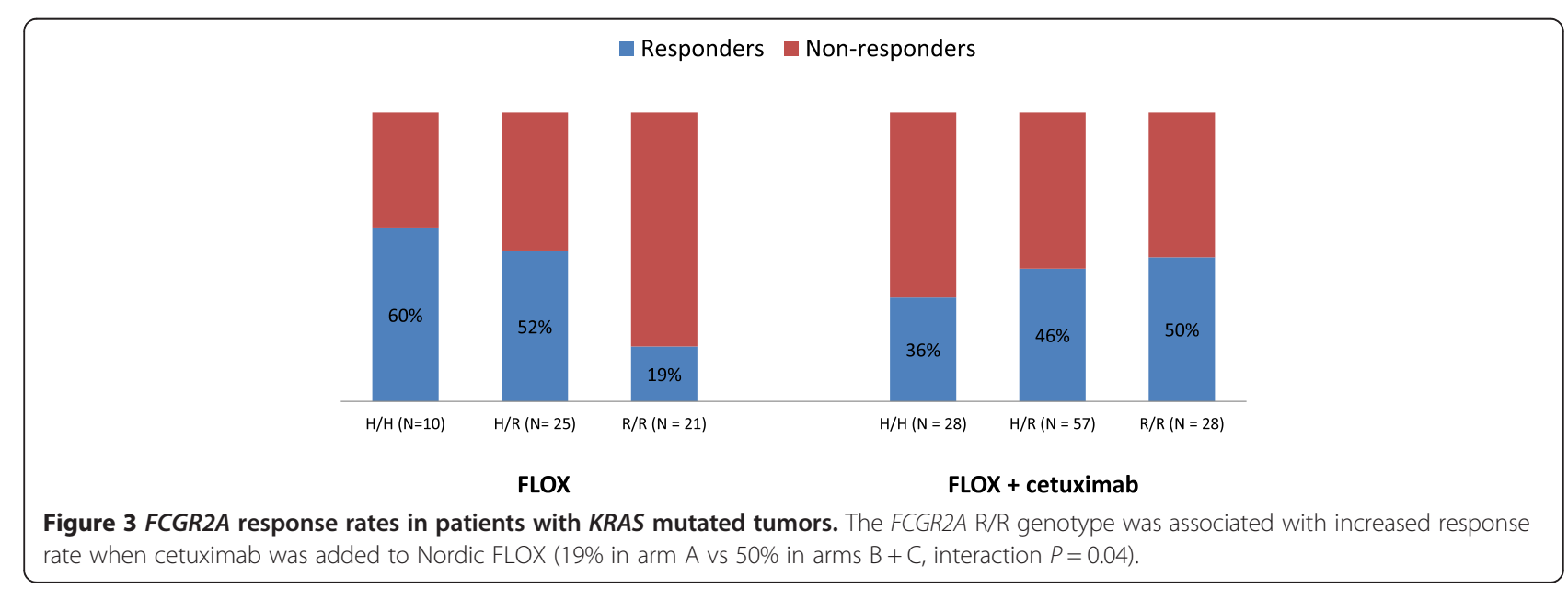


Table 5 Treatment outcome by FCGR3A genotype, KRAS or BRAF mutational status, and therapy received

\begin{tabular}{|c|c|c|c|c|c|c|c|}
\hline & \multicolumn{3}{|l|}{ FLOX } & \multicolumn{3}{|c|}{ FLOX + cetuximab } & \multirow[b]{2}{*}{ Interaction $P$-value } \\
\hline & $F / F$ & $F / V$ & $V / V$ & $F / F$ & $F / V$ & $V / V$ & \\
\hline \multicolumn{8}{|c|}{ KRAS wild-type $(\mathrm{N}=268)$} \\
\hline Number of patients & $N=39$ & $N=38$ & $N=9$ & $N=87$ & $N=73$ & $N=22$ & \\
\hline Response & $44 \%(17 / 39)$ & $45 \%(17 / 38)$ & $56 \%(5 / 9)$ & $48 \%(42 / 87)$ & $51 \%(37 / 73)$ & $55 \%(12 / 22)$ & $0.95^{*}$ \\
\hline PFS & 7.8 & 9.0 & 8.4 & 8.0 & 7.3 & 11.8 & $0.72^{+}$ \\
\hline OS & 23.1 & 20.5 & 25.2 & 17.6 & 25.9 & 20.5 & $0.97^{+}$ \\
\hline \multicolumn{8}{|c|}{ KRAS mutated $(\mathrm{N}=169)$} \\
\hline Number of patients & $N=28$ & $N=21$ & $N=6$ & $N=59$ & $N=46$ & $N=9$ & \\
\hline Response & $36 \%(10 / 28)$ & $48 \%(10 / 21)$ & $50 \%(3 / 6)$ & $51 \%(30 / 59)$ & $39 \%(18 / 46)$ & $33 \%(3 / 9)$ & $0.28^{*}$ \\
\hline PFS & 7.8 & 8.1 & 4.0 & 8.3 & 7.0 & 6.9 & $0.19^{+}$ \\
\hline OS & 18.5 & 24.3 & 17.1 & 21.3 & 17.7 & 16.4 & $0.63^{+}$ \\
\hline \multicolumn{8}{|c|}{$B R A F$ wild-type $(\mathrm{N}=359)$} \\
\hline Number of patients & $N=56$ & $N=47$ & $N=12$ & $N=126$ & $N=94$ & $N=24$ & \\
\hline Response & $45 \%(25 / 56)$ & $49 \%(23 / 47)$ & $50 \%(6 / 12)$ & $52 \%(65 / 126)$ & $48 \%(45 / 94)$ & $63 \%(15 / 24)$ & $0.71^{*}$ \\
\hline PFS & 7.9 & 9.1 & 7.8 & 8.3 & 7.6 & 11.5 & $0.58^{+}$ \\
\hline OS & 23.8 & 23.6 & 19.7 & 20.6 & 22.9 & 20.5 & $0.93^{+}$ \\
\hline \multicolumn{8}{|c|}{ BRAF mutated $(\mathrm{N}=46)$} \\
\hline Number of patients & $N=8$ & $N=7$ & $N=2$ & $N=10$ & $N=14$ & $N=5$ & \\
\hline Response & $13 \%(1 / 8)$ & $14 \%(1 / 7)$ & $50 \%(1 / 2)$ & $20 \%(2 / 10)$ & $29 \%(4 / 14)$ & $0 \%(0 / 5)$ & $0.99^{*}$ \\
\hline PFS & 5.9 & 4.3 & 4.4 & 4.2 & 5.4 & 4.6 & $0.87^{+}$ \\
\hline OS & 9.5 & 9.4 & 5.2 & 10.8 & 8.9 & 10.3 & $0.66^{+}$ \\
\hline
\end{tabular}

"Logistic regression, ${ }^{\dagger}$ Cox proportional hazard model.

immune function in cancer patients may be impaired by the myeloablative effects of chemotherapy which may impair ADCC [39].

Primary tumors in the NORDIC VII study were screened for KRAS exon 2 (codons 12 and 13) mutations. Recent studies have though demonstrated that the selection of patients for anti-EGFR therapy may improve by considering $R A S$ mutations other than KRAS exon 2 mutations (NRAS exons 2, 3, and 4 and KRAS exons 3 and 4) [16-18]. It is expected to find up to $17 \%$ mutations in the KRAS exon 2 wild-type population in the NORDIC VII cohort. We do not expect that the contribution of the additional mutations will considerably alter the outcome of the FCGR polymorphisms. Lack of this data is however a limitation of the present study.

\section{Conclusions}

Patients with KRAS mutated tumors and the FCGR2A $R / R$ genotype responded poorly when treated with chemotherapy only and experienced the most benefit of the addition of cetuximab in terms of response rate. The response rate for the FCGR2A R/R genotype was however not significantly larger than in the other two FCGR2A genotypes $(\mathrm{H} / \mathrm{R}$ and $\mathrm{H} / \mathrm{H})$ in patients treated with Nordic FLOX and cetuximab. Moreover, there was no significant association between any of the FCGR2A genotypes and PFS or OS and the implication of this finding thus remains of uncertain clinical relevance. Many potential associations have been studied, and due to multiplicity a small number of low p-values would be expected to occur by chance even if no true associations exist. Furthermore, we found no significant association between any of the FCGR3A genotypes and response, PFS, or OS. Although our study has a larger sample size than most previously published studies, the sample size in the FCGR subgroups is still too low to obtain sufficient power and larger statistically powered studies to evaluate the significance of the FCGR polymorphisms are needed. Furthermore, the NORDIC VII cohort has limitations for studies of biomarkers predictive of cetuximab effect, as cetuximab did not add significant benefit to the Nordic FLOX regimen. In conclusion, we consider the FCGR2A and FCGR3A polymorphisms not to be currently useful predictive markers of cetuximab efficacy in mCRC.

\section{Competing interests}

The authors declare that they have no competing interests.

\section{Authors' contributions}

AMD and JBK performed the genotyping. JBK analyzed the data and prepared the first draft of the manuscript. ES was involved in the

interpretation of the data and contributed with statistical advice. KMT, TG, TI, CK, MKY, TF were responsible for recruitment of patients, blood sampling and clinical data collection. EHK was responsible for the biobanking. EHK brought the idea and organized the study. All authors read and approved the final manuscript. 


\section{Acknowledgements}

The NORDIC-VII study was supported by Merck-Serono, Darmstadt, Germany and Sanofi-Aventis, Oslo, Norway. This work was supported by the South-Eastern Norway Regional Health Authority.

\section{Author details}

${ }^{1}$ Department of Genetics, Institute for Cancer Research, Oslo University Hospital, Postboks 4953 Nydalen, 0424 Oslo, Norway. ${ }^{2}$ School of Pharmacy, University of Oslo and the Norwegian Institute of Public Health, Oslo, Norway. ${ }^{3}$ Department of Oncology, Oslo University Hospital, Oslo, Norway. ${ }^{4}$ Center for Cancer Treatment, Southern Hospital Trust, Kristiansand, Norway. ${ }^{5}$ Department of Oncology, Aalborg University Hospital, Aalborg, Denmark. ${ }^{6}$ Department of Oncology, Karolinska University Hospital, Stockholm, Sweden.

Received: 6 January 2014 Accepted: 9 May 2014 Published: 19 May 2014

\section{References}

1. Cunningham D, Atkin W, Lenz HJ, Lynch HT, Minsky B, Nordlinger B: Colorectal cancer. Lancet 2010, 375:1030-1047.

2. Tol J, Punt CJ: Monoclonal antibodies in the treatment of metastatic colorectal cancer: a review. Clin Ther 2010, 32:437-453.

3. Karapetis CS, Khambata-Ford S, Jonker DJ, O'Callaghan CJ, Tu D, Tebbutt NC: K-ras mutations and benefit from cetuximab in advanced colorectal cancer. N Engl J Med 2008, 359:1757-1765.

4. Lievre A, Bachet JB, Boige V, Cayre A, Le CD, Buc E: KRAS mutations as an independent prognostic factor in patients with advanced colorectal cancer treated with cetuximab. J Clin Oncol 2008, 26:374-379.

5. Tveit KM, Guren T, Glimelius B, Pfeiffer P, Sorbye H, Pyrhonen S: Phase III trial of cetuximab with continuous or intermittent fluorouracil, leucovorin, and oxaliplatin (Nordic FLOX) versus FLOX alone in first-line treatment of metastatic colorectal cancer: the NORDIC-VII study. J Clin Oncol 2012, 30:1755-1762

6. Maughan TS, Adams RA, Smith CG, Meade AM, Seymour MT, Wilson RH: Addition of cetuximab to oxaliplatin-based first-line combination chemotherapy for treatment of advanced colorectal cancer: results of the randomised phase 3 MRC COIN trial. Lancet 2011, 377:2103-2114.

7. Primrose JN, Falk S, Finch-Jones M, Valle JW, Sherlock D, Hornbuckle J: A randomized clinical trial of chemotherapy compared to chemotherapy in combination with cetuximab in k-RAS wild-type patients with operable metastases from colorectal cancer: the new EPOC study. ASCO Meeting Abstracts 2013, 31:3504

8. Kurai J, Chikumi H, Hashimoto K, Yamaguchi K, Yamasaki A, Sako T: Antibody-dependent cellular cytotoxicity mediated by cetuximab against lung cancer cell lines. Clin Cancer Res 2007, 13:1552-1561.

9. Desjarlais JR, Lazar GA, Zhukovsky EA, Chu SY: Optimizing engagement of the immune system by anti-tumor antibodies: an engineer's perspective. Drug Discov Today 2007, 12:898-910.

10. van Sorge NM, van der Pol WL, van de Winkel JG: FcgammaR polymorphisms: Implications for function, disease susceptibility and immunotherapy. Tissue Antigens 2003, 61:189-202.

11. Weng WK, Levy R: Two immunoglobulin $G$ fragment $C$ receptor polymorphisms independently predict response to rituximab in patients with follicular lymphoma. J Clin Oncol 2003, 21:3940-3947.

12. Cartron G, Dacheux L, Salles G, Solal-Celigny P, Bardos P, Colombat P: Therapeutic activity of humanized anti-CD20 monoclonal antibody and polymorphism in IgG Fc receptor FcgammaRIlla gene. Blood 2002, 99:754-758.

13. Musolino A, Naldi N, Bortesi B, Pezzuolo D, Capelletti M, Missale G: Immunoglobulin $\mathrm{G}$ fragment $\mathrm{C}$ receptor polymorphisms and clinical efficacy of trastuzumab-based therapy in patients with HER-2/neu-positive metastatic breast cancer. J Clin Oncol 2008, 26:1789-1796.

14. Tamura K, Shimizu C, Hojo T, Kashi-Tanaka S, Kinoshita T, Yonemori K: FcgammaR2A and 3A polymorphisms predict clinical outcome of trastuzumab in both neoadjuvant and metastatic settings in patients with HER2-positive breast cancer. Ann Oncol 2011, 22:1302-1307.

15. Therasse P, Arbuck SG, Eisenhauer EA, Wanders J, Kaplan RS, Rubinstein L: New guidelines to evaluate the response to treatment in solid tumors. European Organization for Research and Treatment of Cancer, National Cancer Institute of the United States, National Cancer Institute of Canada. J Natl Cancer Inst 2000, 92:205-216.
16. Douillard JY, Oliner KS, Siena S, Tabernero J, Burkes RF, Barugel MF: Panitumumab-FOLFOX4 treatment and RAS mutations in colorectal cancer. N Engl J Med 2013, 369:1023-1034.

17. Seymour MT, Brown SR, Middleton G, Maughan TF, Richman S, Gwyther S, Lowe C: Panitumumab and irinotecan versus irinotecan alone for patients with KRAS wild-type, fluorouracil-resistant advanced colorectal cancer (PICCOLO): a prospectively stratified randomised trial. Lancet Oncol 2013, 14:749-759.

18. De RW, Claes B, Bernasconi D, De SJ, Biesmans B, Fountzilas G: Effects of KRAS, BRAF, NRAS, and PIK3CA mutations on the efficacy of cetuximab plus chemotherapy in chemotherapy-refractory metastatic colorectal cancer: a retrospective consortium analysis. Lancet Oncol 2010, 11:753-762.

19. Rodriguez S, Gaunt TR, Day IN: Hardy-Weinberg equilibrium testing of biological ascertainment for Mendelian randomization studies. Am J Epidemiol 2009, 169:505-514.

20. Paez D, Pare L, Espinosa I, Salazar J, Del RE, Barnadas A: Immunoglobulin G fragment $C$ receptor polymorphisms and KRAS mutations: are they useful biomarkers of clinical outcome in advanced colorectal cancer treated with anti-EGFR-based therapy? Cancer Sci 2010, 101:2048-2053.

21. Zhang W, Yang D, Harbison C, Khambata-Ford S, Malone DP, Ning Y: FCGR2A H131R and FCGR3A V158F polymorphism status in MCRC patients treated with single-agent cetuximab (IMCL 0144 and CA225045) or with second-line irinotecan plus cetuximab (EPIC): a pooled statistical analysis. ASCO Meeting Abstracts 2011, 29:e14025.

22. Negri FV, Musolino A, Naldi N, Bortesi B, Missale G, Laccabue D: Role of immunoglobulin $\mathrm{G}$ fragment $\mathrm{C}$ receptor polymorphism-mediated antibody-dependant cellular cytotoxicity in colorectal cancer treated with cetuximab therapy. Pharmacogenomics J 2013, 14:14-19.

23. Bibeau F, Lopez-Crapez E, Di FF, Thezenas S, Ychou M, Blanchard F: Impact of Fc\{gamma\}RIla-Fc\{gamma\}RIlla polymorphisms and KRAS mutations on the clinical outcome of patients with metastatic colorectal cancer treated with cetuximab plus irinotecan. J Clin Oncol 2009, 27:1122-1129.

24. Rodriguez J, Zarate R, Bandres E, Boni V, Hernandez A, Sola JJ: Fc gamma receptor polymorphisms as predictive markers of Cetuximab efficacy in epidermal growth factor receptor downstream-mutated metastatic colorectal cancer. Eur J Cancer 2012, 48:1774-1780.

25. Etienne-Grimaldi MC, Bennouna J, Formento JL, Douillard JY, Francoual M, Hennebelle I: Multifactorial pharmacogenetic analysis in colorectal cancer patients receiving 5-fluorouracil-based therapy together with cetuximabirinotecan. Br J Clin Pharmacol 2012, 73:776-785.

26. Calemma R, Ottaiano A, Trotta AM, Nasti G, Romano C, Napolitano M: Fc gamma receptor Illa polymorphisms in advanced colorectal cancer patients correlated with response to anti-EGFR antibodies and clinical outcome. J Transl Med 2012, 10:232

27. Zhang W, Azuma M, Lurje G, Gordon MA, Yang D, Pohl A: Molecular predictors of combination targeted therapies (cetuximab, bevacizumab) in irinotecan-refractory colorectal cancer (BOND-2 study). Anticancer Res 2010, 30:4209-4217.

28. Dahan L, Norguet E, Etienne-Grimaldi MC, Formento JL, Gasmi M, Nanni I: Pharmacogenetic profiling and cetuximab outcome in patients with advanced colorectal cancer. BMC Cancer 2011, 11:496.

29. Pander J, Gelderblom H, Antonini NF, Tol J, van Krieken JH, van der ST: Correlation of FCGR3A and EGFR germline polymorphisms with the efficacy of cetuximab in KRAS wild-type metastatic colorectal cancer. Eur $J$ Cancer 2010, 46:1829-1834

30. Zhang W, Gordon M, Schultheis AM, Yang DY, Nagashima F, Azuma M: FCGR2A and FCGR3A polymorphisms associated with clinical outcome of epidermal growth factor receptor expressing metastatic colorectal cancer patients treated with single-agent cetuximab. J Clin Oncol 2007, 25:3712-3718.

31. Lurje G, Nagashima F, Zhang W, Yang D, Chang HM, Gordon MA: Polymorphisms in cyclooxygenase-2 and epidermal growth factor receptor are associated with progression-free survival independent of K-ras in metastatic colorectal cancer patients treated with single-agent cetuximab. Clin Cancer Res 2008, 14:7884-7895.

32. Graziano F, Ruzzo A, Loupakis F, Canestrari E, Santini D, Catalano V Pharmacogenetic profiling for cetuximab plus irinotecan therapy in patients with refractory advanced colorectal cancer. J Clin Oncol 2008, 26:1427-1434.

33. Park SJ, Hong YS, Lee JL, Ryu MH, Chang HM, Kim KP: Genetic polymorphisms of FcgammaRlla and FcgammaRIlla are not predictive of clinical outcomes after cetuximab plus irinotecan chemotherapy in patients with metastatic colorectal cancer. Oncology 2012, 82:83-89. 
34. Geva R, Jensen BV, Fountzilas G, Yoshino T, Paez D, Montagut C: An international consortium study in chemorefractory metastatic colorectal cancer (mCRC) patients (pts) to assess the impact of FCGR polymorphisms on cetuximab efficacy. ASCO Meeting Abstracts 2011, 29:3528.

35. Correale P, Marra M, Remondo C, Migali C, Misso G, Arcuri FP: Cytotoxic drugs up-regulate epidermal growth factor receptor (EGFR) expression in colon cancer cells and enhance their susceptibility to EGFR-targeted antibody-dependent cell-mediated-cytotoxicity (ADCC). Eur J Cancer 2010, 46:1703-1711.

36. Schlaeth M, Berger S, Derer S, Klausz K, Lohse S, Dechant M: Fc-engineered EGF-R antibodies mediate improved antibody-dependent cellular cytotoxicity (ADCC) against KRAS-mutated tumor cells. Cancer Sci 2010, 101:1080-1088.

37. Clynes RA, Towers TL, Presta LG, Ravetch JV: Inhibitory Fc receptors modulate in vivo cytotoxicity against tumor targets. Nat Med 2000, 6:443-446.

38. Kono K, Takahashi A, Ichihara F, Sugai H, Fujii H, Matsumoto Y: Impaired antibody-dependent cellular cytotoxicity mediated by herceptin in patients with gastric cancer. Cancer Res 2002, 62:5813-5817.

39. de Souza AP, Bonorino C: Tumor immunosuppressive environment: effects on tumor-specific and nontumor antigen immune responses. Expert Rev Anticancer Ther 2009, 9:1317-1332.

doi:10.1186/1471-2407-14-340

Cite this article as: Kjersem et al:: FCGR2A and FCGR3A polymorphisms and clinical outcome in metastatic colorectal cancer patients treated with first-line 5-fluorouracil/folinic acid and oxaliplatin +/- cetuximab. BMC Cancer 2014 14:340.

\section{Submit your next manuscript to BioMed Central and take full advantage of:}

- Convenient online submission

- Thorough peer review

- No space constraints or color figure charges

- Immediate publication on acceptance

- Inclusion in PubMed, CAS, Scopus and Google Scholar

- Research which is freely available for redistribution 